\title{
Exact stability test of neutral delay differential equations via a rough estimation of the testing integral
}

\author{
Qi Xu • Zaihua Wang
}

Received: 30 July 2013 / Revised: 14 November 2013 / Accepted: 19 November 2013 / Published online: 10 December 2013

(C) Springer-Verlag Berlin Heidelberg 2013

\begin{abstract}
In this paper, we study the problem of stability test of neutral delay differential equations. Firstly we prove that the Mikhailov stability criterion and its equivalent integral form for ordinary differential equations hold for neutral delay differential equations in general form. The criteria are simple in form that is easy for numerical implementation. However, the criteria are characterized by an auxiliary function associated with the characteristic function, not by the characteristic function itself. To reduce the computational complexity, we further prove that a Mikhailov-type criterion in terms of the characteristic function holds. With this new criterion, the stability of a given neutral delay differential equation can be tested with a rough estimation of the testing integral. Thus, the computational complexity and computational cost can be greatly reduced. As two applications of these criteria, we firstly propose a numerical scheme for calculating the rightmost characteristic $\operatorname{root}(\mathrm{s})$ as well as the characteristic roots other than the rightmost roots of a given neutral delay differential equation, demonstrated with two examples. Then we derive a graphical stability criterion. With this graphical stability criterion, it is not required to know the exact curve of the Nyquist plot, but just to know whether the Nyquist plot encircles the origin of the complex plane or not.
\end{abstract}

Keywords Time delay - Delay differential equation . Stability criterion $\cdot$ Stability test $\cdot$ Numerical integration

\footnotetext{
Q. Xu $\cdot \mathrm{Z}$. Wang $(\varangle)$

State Key Laboratory of Mechanics and Control of Mechanical Structures, Nanjing University of Aeronautics and Astronautics, Nanjing 210016, China

e-mail: zhwang@nuaa.edu.cn

Q. Xu

e-mail: xuqi@nuaa.edu.cn
}

\section{Introduction}

Delay differential equations of neutral type (NDDEs for short) are differential equations that involve a single delay or multiple delays in the highest-order derivative, and they are encountered frequently in different engineering applications [1-7]. For example, in controlling the vibration of a beam, a NDDE delayed resonator was proposed on the basis of delayed acceleration feedback [3], this technique utilizes acceleration sensor for its advantage in high frequency-low amplitude situation, the delayed signal of acceleration sensor leads to NDDE. Another example of NDDE comes from the problem of reducing the sway of container crane by using a delayed nonlinear controller [4,5]. Having been successfully installed on a huge industrial container crane, this delayed control can effectively reduce the cargo sway and therefore greatly improve the crane productivity. NDDEs are also encountered in the applications of real-time dynamic substructuring method [6]. In studying the dynamic response to complex structures, this method replaces part of the physical structure with a numerical model, and a time delay naturally arises when combining both numerical and remained part of the physical substructure by using actuators. In addition, a nonlinear NDDE was used to model transmission line oscillator, which can produce chaotic high-frequency output and demonstrate rich dynamics induced by the delay [7]. Numerical analysis shows that the NDDE affects certain dynamics such as the convergence rates of the fold points to the homoclinic tangencies.

For autonomous DDEs, complicated nonlinear dynamics is possible if the system undergoes a number of bifurcations with the change of some parameter. Thus, stability analysis is one of the major topics about DDEs in the literature. Compared with delay differential equations of retarded type (RDDEs for short) that have no delayed derivative terms of 
the highest order, NDDEs exhibit some peculiarities from the view point of stability analysis. For example, a RDDE always has a finite number of characteristic roots in the right-half complex plane only, but a NDDE may have an infinite number of characteristic roots in the right-half complex plane. In addition, the asymptotical stability of a RDDE is guaranteed if all the characteristic roots have negative real parts, but a NDDE may not be asymptotically stable when such a condition holds, because the infinite number of roots may have accumulation points on the imaginary axis. Under some additional conditions placed on the coefficients of the highest-order delayed terms, the asymptotical stability of a NDDE is guaranteed by the condition "all the characteristic roots have negative real parts" $[1,2]$. In this case, many stability criteria for RDDEs can be generalized to NDDEs. For example, the Nyquist plots can be used to test the stability of RDDEs [8,9] and NDDEs [10]. This is also true for the method of stability switches, which was proposed to determine the stable intervals of a parameter including delay or feedback gain [1]. Together with D-subdivision method, this method can also demonstrate the stability maps against multiple parameters of RDDEs [14] or NDDEs [15]. The Hassard theorem [11], a different version of Stepan Theorem [12] that gives an explicit formula for calculating the number of the unstable characteristic roots of RDDEs, has been extended to NDDEs[13]. The semi-discretization method for RDDEs [16] has been generalized to NDDEs [17]. This method discretizes the delayed terms while remained actual time terms are unchanged, and can be used to study periodic continuous DDEs. It is referred to see the monograph [18] for a detailed introduction of the semi-discretization method with applications to stability analysis. In addition, the rightmost characteristic root(s) tells not only the system stability, but also the convergence speed of the time history of the DDE to the target state. A numerical scheme [19] for calculating the rightmost characteristic roots of RDDEs is extended to NDDEs [20]. Following this method, an auxiliary function associated with the characteristic function is firstly constructed by using the Lambert $\mathrm{W}$ function, then the rightmost roots can be found by properly using the Newton-Raphson iteration method. Using a linear multi-step method, a MATLAB package called DDEBIFTOOL [21] dealing with RDDEs has been extended to NDDEs [22]. Within this frame, one first approximates the roots of linearized characteristic equations, then use Newton iteration to correct the approximate roots. This package has found many applications in the literature. Usually, the available numerical methods uses approximation that reduces an infinite-dimensional delay differential equation to a finitedimensional ordinary differential equation. In this way, calculation accuracy in stability test depends on approximation accuracy.

Mikhailov formula is an analytical stability criterion derived on the basis of Argument Principle for complex functions. It states that the asymptotical stability of a system is justified if the change of the argument of the characteristic function along the positive imaginary axis equals to $n \pi / 2$, where $n$ is the order of the system. This formula has been proved true for RDDEs [23]. Although beautiful in form, this criterion is actually not convenient in stability analysis. In applications, its equivalent integral form is much very flexible and effective, and it has been extended for checking whether a delay-fractional system has characteristic roots with negative real parts only, and for calculating the rightmost characteristic roots [24]. The main objective of this paper is to prove that Mikhailov formula and its equivalent form hold for NDDEs. The stability criteria can be characterized by an auxiliary function associated with the characteristic function or by the characteristic function directly. Unlike the numerical methods available in the literature that use proper approximation of the NDDEs or their characteristic functions with high accuracy, the new proposed stability criteria enable us to test the stability of a given NDDE via a rough estimation of the testing integral.

The rest of the paper is organized as follows. Firstly in Sect. 2, we present the main results with proofs, including a Mikhailov-type stability criterion and its equivalent integral form in terms of an axillary function, and a stability criterion in terms of the characteristic function. Then in Sect. 3, based on the proposed stability criteria, we propose a numerical scheme for the calculation of the rightmost characteristic roots and the other characteristic roots, and in Sect. 4, we give two examples for demonstration. As another application of the proposed Mikhailov-type stability criteria, a graphical stability criterion is also derived in Sect. 5. Finally in Sect. 6, we draw some concluding remarks from the investigation.

\section{Mikhailov-type stability criteria}

Let us consider linear NDDEs described by

$$
\dot{x}(t)+\sum_{i=1}^{m} N_{i} \dot{x}\left(t-\tau_{i}\right)=A x(t)+\sum_{i=1}^{m} B_{i} x\left(t-\tau_{i}\right)
$$

where $x \in \mathbb{R}^{n}, A, B_{i}, N_{i} \in \mathbb{R}^{n \times n}$, and at least one $N_{k} \neq 0$ for some $k=1,2, \ldots, m$. The characteristic equation of Eq. (1) is in the form $f(\lambda)=0$ with

$$
f(\lambda)=\lambda^{n}+\sum_{i=0}^{n} \alpha_{i}\left(\mathrm{e}^{-\lambda \tau_{1}}, \ldots, \mathrm{e}^{-\lambda \tau_{m}}\right) \lambda^{n-i}
$$

where $\alpha_{i}\left(z_{1}, \ldots, z_{m}\right),(i=0,1, \ldots, n)$, are real polynomials with respect to $z_{1}=\mathrm{e}^{-\lambda \tau_{1}}, \ldots, z_{m}=\mathrm{e}^{-\lambda \tau_{m}}$. The trivial solution $x=0$ of Eq. (1) is asymptotically stable if and only if the characteristic roots lie in the left half complex plane and are uniformly bounded away from the imaginary axis [1]. This is true if the function $\alpha_{0}\left(\mathrm{e}^{-\lambda \tau_{1}}, \ldots, \mathrm{e}^{-\lambda \tau_{m}}\right)$ satisfies 
Fig. 1 The integral contour $C$

$\sup _{\Re(\lambda)>0}\left|\alpha_{0}\left(\mathrm{e}^{-\lambda \tau_{1}}, \ldots, \mathrm{e}^{-\lambda \tau_{m}}\right)\right|<1$

where $\Re(z)$ is the real part of complex number $z$. Condition (3) ensures that the characteristic function $f(\lambda)$ has only finite number of roots located in the right half complex plane and has no roots with accumulation points on the imaginary axis. Without loss of generality, let the coefficients of the nonzero terms of $\alpha_{0}\left(z_{1}, \ldots, z_{m}\right)$ be denoted by $\beta_{1}, \beta_{2}, \ldots, \beta_{k} \in$ $\mathbb{R}$, then one has

$\sup _{\substack{\Re(\lambda)>0 \\|\lambda| \rightarrow \infty}}\left|\alpha_{0}\left(\mathrm{e}^{-\lambda \tau_{1}}, \ldots, \mathrm{e}^{-\lambda \tau_{m}}\right)\right|=\sum_{j=1}^{k}\left|\beta_{j}\right|$

If condition (3) is satisfied, the stability can be tested by calculating the number $\mathcal{N}$ of characteristic roots with nonnegative real parts, or by calculating the real part $\sigma$ of the rightmost characteristic $\operatorname{root}(\mathrm{s})$. The trivial solution $x=0$ of Eq. (1) is asymptotically stable if and only if $\mathcal{N}=0$ or $\sigma<0$.

Assume that $f(\lambda)$ has no roots on the imaginary axis, and let $\Delta_{C} \arg f(\lambda)$ denote the change in the argument of $f(\lambda)$ over the contour $C$ shown in Fig. 1, then the number $\mathcal{N}$ of the characteristic roots in the open right-half complex plane of $\lambda$ can be calculated with

$\mathcal{N}=\lim _{R \rightarrow+\infty} \frac{\Delta_{C} \arg (f(\lambda))}{2 \pi}$

where

$C_{1}: \quad \lambda=R \mathrm{e}^{\mathrm{i} \theta}, \quad \theta$ increases from $-\frac{\pi}{2}$ to $\frac{\pi}{2} ;$

$C_{2}: \quad \lambda=\mathrm{i} \omega, \quad \omega$ decreases from $R$ to $-R$.

Under the assumption (3), $1+\alpha_{0}\left(\mathrm{e}^{-\lambda \tau_{1}}, \ldots, \mathrm{e}^{-\lambda \tau_{m}}\right) \neq 0$ holds for any $\lambda \in \mathbb{C}$. Hence, the auxiliary function $W(\lambda)$ defined by

$W(\lambda)=\frac{f(\lambda)}{1+\alpha_{0}\left(z_{1}, \ldots, z_{m}\right)}=\lambda^{n}+\sum_{i=1}^{n} \xi_{i}\left(z_{1}, \ldots, z_{m}\right) \lambda^{n-i}$ is well-defined, where $z_{1}=\mathrm{e}^{-\lambda \tau_{1}}, \ldots, z_{m}=\mathrm{e}^{-\lambda \tau_{m}}$, and each $\xi_{i}\left(z_{1}, \ldots, z_{m}\right)$ is analytic with respect to $z_{1}, \ldots, z_{m}$. Obviously, one has

$\mathcal{N}=\lim _{R \rightarrow+\infty} \frac{\Delta_{C} \arg (W(\lambda))}{2 \pi}$

Theorem 1 Assume that the characteristic function $f(\lambda)$, defined by Eq. (2), of a NDDE has no roots on the imaginary axis, and condition (3) holds. Let $W(\lambda)$ be the function defined by (6), then $\mathcal{N}=0$ holds if and only if

$\int_{0}^{+\infty} \Re\left(\frac{W^{\prime}(\mathrm{i} \omega)}{W(\mathrm{i} \omega)}\right) \mathrm{d} \omega=\frac{n \pi}{2}$

where the integral is called "testing integral".

Proof In fact, on the right-half complex plane with $\lambda \in \mathbb{C}$ satisfying $\Re(\lambda)>0$ and $|\lambda| \rightarrow+\infty$, one has

$\frac{W(\lambda)-\lambda^{n}}{\lambda^{n}} \rightarrow 0$

Then for $\theta \in\left[-\frac{\pi}{2}, \frac{\pi}{2}\right]$ and $R \gg 1$, one has

$$
\begin{aligned}
\Delta_{C_{1}} \arg (W(\lambda)) & =\Delta_{C_{1}} \arg \left(\lambda^{n}\right)+\Delta_{C_{1}} \arg \left(1+\frac{W(\lambda)-\lambda^{n}}{\lambda^{n}}\right) \\
& \approx \Delta_{C_{1}} \arg \left(R^{n} \mathrm{e}^{n \mathrm{i} \theta}\right)+\Delta_{C_{1}} \arg (1)
\end{aligned}
$$

Hence, as $R \rightarrow+\infty$, one has

$$
\Delta_{C_{1}} \arg (W(\lambda)) \rightarrow 0+n \frac{\pi}{2}-\left(-n \frac{\pi}{2}\right)=n \pi
$$

In addition, when $R \rightarrow+\infty$, one has

$$
\begin{aligned}
\Delta_{C_{2}} \arg (W(\lambda)) & =\left.\arg (W(\mathrm{i} \omega))\right|_{\omega=R} ^{\omega=-R} \\
& \rightarrow-\left.\arg (W(\mathrm{i} \omega))\right|_{\omega \rightarrow-\infty} ^{\omega \rightarrow+\infty} \\
& =-\left.2 \arg (W(\mathrm{i} \omega))\right|_{\omega=0} ^{\omega \rightarrow+\infty}
\end{aligned}
$$

It follows that

$$
\begin{aligned}
\mathcal{N} & =\frac{n \pi-\left.2 \arg (W(\mathrm{i} \omega))\right|_{\omega=0} ^{\omega \rightarrow+\infty}}{2 \pi} \\
& =\frac{n}{2}-\left.\frac{1}{\pi} \arg (W(\mathrm{i} \omega))\right|_{\omega=0} ^{\omega \rightarrow+\infty}
\end{aligned}
$$

Moreover, it is easy to show that

$\frac{\mathrm{d}}{\mathrm{d} \omega} \arg (W(\mathrm{i} \omega))=\mathfrak{R}\left(\frac{W^{\prime}(\mathrm{i} \omega)}{W(\mathrm{i} \omega)}\right)$

Therefore, using Newton-Leibnitz formula leads to

$\int_{0}^{+\infty} \Re\left(\frac{W^{\prime}(\mathrm{i} \omega)}{W(\mathrm{i} \omega)}\right) \mathrm{d} \omega=(n-2 \mathcal{N}) \cdot \frac{\pi}{2}$

where the testing integral on the left side always converges due to

$\Re\left(\frac{W^{\prime}(\mathrm{i} \omega)}{W(\mathrm{i} \omega)}\right)=O\left(\frac{1}{\omega^{2}}\right), \quad(\omega \gg 1)$ 
Hence, $\mathcal{N}=0$ holds if and only if Eq. (8) holds. This completes the proof.

Corollary 1 Under the assumptions of Theorem 1, Eq. (8) is true if and only if there is a sufficiently large $T>0$ such that

$\int_{0}^{T} \Re\left(\frac{W^{\prime}(\mathrm{i} \omega)}{W(\mathrm{i} \omega)}\right) \mathrm{d} \omega>\frac{(n-1) \pi}{2}$

Proof If Eq. (8) holds, namely $\mathcal{N}=0$, then for any given $\varepsilon>0$, there is a $T_{0}$ such that for all $T>T_{0}$ one has

$\left|\int_{0}^{T} \Re\left(\frac{W^{\prime}(\mathrm{i} \omega)}{W(\mathrm{i} \omega)}\right) \mathrm{d} \omega-\frac{n \pi}{2}\right|<\varepsilon$

In particular, for $\varepsilon=\pi / 2$, there is a $T_{0}$ such that for all $T>T_{0}$ one has

$\left|\int_{0}^{T} \mathfrak{R}\left(\frac{W^{\prime}(\mathrm{i} \omega)}{W(\mathrm{i} \omega)}\right) \mathrm{d} \omega-\frac{n \pi}{2}\right|<\frac{\pi}{2}$

It follows that (12) holds for all $T>T_{0}$.

Conversely, if on the contrary (12) does not hold, namely if for any $T>0$ one has

$\int_{0}^{T} \Re\left(\frac{W^{\prime}(\mathrm{i} \omega)}{W(\mathrm{i} \omega)}\right) \mathrm{d} \omega \leq \frac{(n-1) \pi}{2}$

then it follows that

$\mathcal{N}=\frac{n}{2}-\frac{1}{\pi} \int_{0}^{+\infty}\left(\frac{W^{\prime}(\mathrm{i} \omega)}{W(\mathrm{i} \omega)}\right) \mathrm{d} \omega \geq \frac{n}{2}-\frac{1}{\pi} \frac{(n-1) \pi}{2}=\frac{1}{2}$

Hence $\mathcal{N} \geq 1$ because $\mathcal{N}$ is an integer. It means that the characteristic function has at least one roots with positive real part. The proof is completed.

Corollary 1 implies that in the stability test of a given NDDE, it is not necessary to calculate the exact value of the testing integral, but just to find an estimation of the testing integral with a properly chosen large $T$. This reduces the computational cost in the stability test considerably. In real applications, it is preferable if the stability test is carried out by using the characteristic function $f(\lambda)$ directly. To see such a case, let us firstly note that for any $T>0$, the argument rule gives

$$
\begin{aligned}
& \int_{0}^{T} \Re\left(\frac{W^{\prime}(\mathrm{i} \omega)}{W(\mathrm{i} \omega)}\right) \mathrm{d} \omega-\int_{0}^{T} \Re\left(\frac{f^{\prime}(\mathrm{i} \omega)}{f(\mathrm{i} \omega)}\right) \mathrm{d} \omega \\
& \quad=\left.\arg (W(\mathrm{i} \omega))\right|_{\omega=0} ^{\omega=T}-\left.\arg (f(\mathrm{i} \omega))\right|_{\omega=0} ^{\omega=T} \\
& =-\left.\arg \left(1+\alpha_{0}\left(\mathrm{e}^{-\mathrm{i} \omega \tau_{1}}, \ldots, \mathrm{e}^{-\mathrm{i} \omega \tau_{m}}\right)\right)\right|_{\omega=0} ^{\omega=T} \\
& =-\arg \left(1+\alpha_{0}\left(\mathrm{e}^{-\mathrm{i} T \tau_{1}}, \ldots, \mathrm{e}^{-\mathrm{i} T \tau_{m}}\right)\right)
\end{aligned}
$$

Unlike in Corollary 1 where the testing integral for $W(\lambda)$ converges, namely as $T \rightarrow+\infty$, the limit

$\int_{0}^{T} \Re\left(\frac{W^{\prime}(\mathrm{i} \omega)}{W(\mathrm{i} \omega)}\right) \mathrm{d} \omega \rightarrow \int_{0}^{+\infty} \Re\left(\frac{W^{\prime}(\mathrm{i} \omega)}{W(\mathrm{i} \omega)}\right) \mathrm{d} \omega$

exits, here in the present case the testing integral for $f(\lambda)$ does not converge because the difference between the two integrals, given by Eq. (13), is not convergent as $\omega=T \rightarrow$ $+\infty$. However, the following lemma holds.

Lemma 1 For the characteristic function $f(\lambda)$, there is a number $T_{0}>0$ such that for all $T>T_{0}$, one has

$$
\int_{0}^{T} \Re\left(\frac{f^{\prime}(\mathrm{i} \omega)}{f(\mathrm{i} \omega)}\right) \mathrm{d} \omega \in\left(\frac{(n-2 \mathcal{N}-1) \pi}{2}, \frac{(n-2 \mathcal{N}+1) \pi}{2}\right)
$$

Proof In fact, under the condition (3), for any $T>0$, the real part of $1+\alpha_{0}\left(\mathrm{e}^{-\mathrm{i} T \tau_{1}}, \ldots, \mathrm{e}^{-\mathrm{i} T \tau_{m}}\right)$ must be positive, which means that the argument in absolute of $1+$ $\alpha_{0}\left(\mathrm{e}^{-\mathrm{i} T \tau_{1}}, \ldots, \mathrm{e}^{-\mathrm{i} T \tau_{m}}\right)$ is less than $\pi / 2$. Moreover, because $1+\alpha_{0}\left(\mathrm{e}^{-\mathrm{i} \omega \tau_{1}}, \ldots, \mathrm{e}^{-\mathrm{i} \omega \tau_{m}}\right)$ is continuous and periodic with respect to $\omega$ when all the time delays are commensurate, or continuous and quasi-periodic with respect to $\omega$ when the delays are incommensurate, there must be a constant $0<\gamma<\pi / 2$ such that

$\left|\arg \left(1+\alpha_{0}\left(\mathrm{e}^{-\mathrm{i} \omega \tau_{1}}, \ldots, \mathrm{e}^{-\mathrm{i} \omega \tau_{m}}\right)\right)\right| \leq \frac{\pi}{2}-\gamma$

For such a constant $\gamma$, there is a constant $T_{0}>0$ such that for all $T>T_{0}$, one has

$\left|\int_{0}^{T} \Re\left(\frac{W^{\prime}(\mathrm{i} \omega)}{W(\mathrm{i} \omega)}\right) \mathrm{d} \omega-(n-2 \mathcal{N}) \frac{n \pi}{2}\right|<\gamma$

because Eq. (10) holds. It follows that for $T>T_{0}$ one has

$$
\begin{aligned}
\int_{0}^{T} \Re\left(\frac{f^{\prime}(\mathrm{i} \omega)}{f(\mathrm{i} \omega)}\right) \mathrm{d} \omega & >\left(\frac{(n-2 \mathcal{N}) \pi}{2}-\gamma\right)+\left(-\frac{\pi}{2}+\gamma\right) \\
& =\frac{(n-2 \mathcal{N}-1) \pi}{2}
\end{aligned}
$$

and

$$
\begin{aligned}
\int_{0}^{T} \Re\left(\frac{f^{\prime}(\mathrm{i} \omega)}{f(\mathrm{i} \omega)}\right) \mathrm{d} \omega & <\left(\frac{(n-2 \mathcal{N}) \pi}{2}+\gamma\right)+\left(\frac{\pi}{2}-\gamma\right) \\
& =\frac{(n-2 \mathcal{N}+1) \pi}{2}
\end{aligned}
$$

This completes the proof.

Now, we are ready to prove the key observation of this paper. With this stability criterion, the computational complexity in the stability test can be even more significantly reduced. 
Theorem 2 Under the assumptions of Theorem 1, Eq. (8) is true if and only if there is a sufficiently large $T>0$ such that

$\int_{0}^{T} \Re\left(\frac{f^{\prime}(\mathrm{i} \omega)}{f(\mathrm{i} \omega)}\right) \mathrm{d} \omega>\frac{(n-1) \pi}{2}$

Proof If $\mathcal{N}=0$, then obviously (17) holds due to (15). Conversely, if for any sufficiently large $T>0$ one has

$\int_{0}^{T} \Re\left(\frac{f^{\prime}(\mathrm{i} \omega)}{f(\mathrm{i} \omega)}\right) \mathrm{d} \omega<\frac{(n-1) \pi}{2}$

then $\mathcal{N} \geq 1$ due to (16). Thus, $\mathcal{N}=0$ holds if and only if (17) is true.

\section{A numerical method for calculating the rightmost characteristic $\operatorname{root}(\mathrm{s})$}

Lots of well-developed numerical methods are available for calculating the approximation of the testing integral over a finite interval, thus the stability test of a given NDDE can be carried out effectively by using Corollary 1 or Theorem 2 . By repeatedly use of this criterion, the stable intervals and unstable intervals within a given range of the delay or some other parameter can be obtained. Moreover, Lemma 1 and Theorem 2 can also be used to calculate the rightmost characteristic $\operatorname{root}(\mathrm{s})$ as well the other characteristic roots in a simple way.

Let $\sigma$ be the abscissa of a given NDDE, defined by

$\sigma=\max \{\Re(\lambda): f(\lambda)=0\}$

where $f(\lambda)$ is the characteristic function in the form of Eq. (2). The number $\sigma$ must be a finite number because all the characteristic roots of the NDDE stay in a strip parallel to the imaginary axis. Under the condition (3), the NDDE is asymptotically stable if and only if $\sigma<0$. The large of the negative $\sigma$ in absolute, the better stability of the system. In this case, $f(\lambda)=0$ has no roots on $\Re(\lambda)=\sigma$ with the accumulation points on the infinity of the imaginary axis. Consequently $f(\sigma+\lambda)=0$ has no roots with the accumulation points on the infinity of the imaginary axis and also no infinite number of roots on the right half plane[1], providing that

$\sup _{\substack{\Re(\lambda)>0 \\|\lambda| \rightarrow \infty}}\left|\alpha_{0}\left(\mathrm{e}^{-(\sigma+\lambda) \tau_{1}}, \ldots, \mathrm{e}^{-(\sigma+\lambda) \tau_{m}}\right)\right|<1$

Hence, for $f(\xi+\lambda)=0$ with $\xi$ close enough to $\sigma$, one has

$\sup _{\Re(\lambda)>0}\left|\alpha_{0}\left(\mathrm{e}^{-(\xi+\lambda) \tau_{1}}, \ldots, \mathrm{e}^{-(\xi+\lambda) \tau_{m}}\right)\right|<1$

which holds true if $\sigma>0$.

Now, the rightmost root(s) can be found out by utilizing the above criteria to test whether or not the roots of $f(\xi+\lambda)=0$ have negative real parts only, if $\xi$ is close enough to $\sigma$, in three steps.

The first step is to find two estimation numbers $\bar{R}, R$ with $\underline{R}<\bar{R}$ such that $\underline{R}<\sigma<\bar{R}$. For any given $\xi, \eta,(\xi<\eta<$ $0)$, one has

$$
\begin{aligned}
& \sup _{\substack{\Re(\lambda)>0 \\
|\lambda| \rightarrow \infty}}\left|\alpha_{0}\left(\mathrm{e}^{-(\eta+\lambda) \tau_{1}}, \ldots, \mathrm{e}^{-(\eta+\lambda) \tau_{m}}\right)\right| \\
& <\sup _{\substack{\Re(\lambda)>0 \\
|\lambda| \rightarrow \infty}}\left|\alpha_{0}\left(\mathrm{e}^{-(\xi+\lambda) \tau_{1}}, \ldots, \mathrm{e}^{-(\xi+\lambda) \tau_{m}}\right)\right|
\end{aligned}
$$

Thus, the two numbers $\bar{R}, \underline{R}$ can be obtained by checking

$$
\left\{\begin{array}{l}
\sup _{\substack{\Re(\lambda)>0 \\
|\lambda| \rightarrow \infty}}\left|\alpha_{0}\left(\mathrm{e}^{-(\underline{R}+\lambda) \tau_{1}}, \ldots, \mathrm{e}^{-(\underline{R}+\lambda) \tau_{m}}\right)\right|<1 \\
\int_{0}^{T} \Re\left(\frac{W^{\prime}(\underline{R}+\mathrm{i} \omega)}{W(\underline{R}+\mathrm{i} \omega)}\right) \mathrm{d} \omega<\frac{(n-1) \pi}{2} \\
\int_{0}^{T} \Re\left(\frac{W^{\prime}(\bar{R}+\mathrm{i} \omega)}{W(\bar{R}+\mathrm{i} \omega)}\right) \mathrm{d} \omega>\frac{(n-1) \pi}{2}
\end{array}\right.
$$

or more directly by checking

$$
\left\{\begin{array}{l}
\sup _{\substack{\Re(\lambda)>0 \\
|\lambda| \rightarrow \infty}}\left|\alpha_{0}\left(\mathrm{e}^{-(\underline{R}+\lambda) \tau_{1}}, \ldots, \mathrm{e}^{-(\underline{R}+\lambda) \tau_{m}}\right)\right|<1 \\
\int_{0}^{T} \Re\left(\frac{f^{\prime}(\underline{R}+\mathrm{i} \omega)}{f(\underline{R}+\mathrm{i} \omega)}\right) \mathrm{d} \omega<\frac{(n-1) \pi}{2} \\
\int_{0}^{T} \Re\left(\frac{f^{\prime}(\bar{R}+\mathrm{i} \omega)}{f(\bar{R}+\mathrm{i} \omega)}\right) \mathrm{d} \omega>\frac{(n-1) \pi}{2}
\end{array}\right.
$$

The interval $[\underline{R}, \bar{R}]$ can be narrowed by repeated use of the above procedure.

The second step is to find an estimation of the rightmost $\operatorname{root}(\mathrm{s})$. When $\bar{R}-\underline{R}$ is small enough, taking $\alpha_{0} \in(R, \bar{R})$ as an initial guess of $\sigma$, an estimation $\omega_{0}$ of the imaginary part $\omega$ of the rightmost characteristic $\operatorname{root}(\mathrm{s})$ can be found numerically from $f\left(\sigma_{0}+\mathrm{i} \omega\right) \approx 0$. Each real root $\omega$ of $f\left(\sigma_{0}+\right.$ $\mathrm{i} \omega) \approx 0$ corresponds to a possible characteristic root (finite and isolated) on the line $\Re(\lambda)=\sigma$.

The final step is to refine the above estimation. Starting from $\sigma_{0}+\mathrm{i} \omega_{0}$, the rightmost characteristic $\operatorname{root}(\mathrm{s})$ can be determined by using the Newton-Raphson iteration method with a few number of iterations:

$\lambda_{i+1}=\lambda_{i}-\frac{f\left(\lambda_{i}\right)}{f^{\prime}\left(\lambda_{i}\right)}, \quad(i=0,1,2, \ldots)$

For a given tolerance $\varepsilon>0$, the iteration procedure is stopped if $\left|\lambda_{k+1}-\lambda_{k}\right|<\varepsilon$.

It is worthy of mentioning that the numerical scheme introduced above works also for calculating the characteristic roots besides the rightmost $\operatorname{root}(\mathrm{s})$. In fact, according to Lemma 1 , for sufficiently large $T>0$, the integral

$\int_{0}^{T} \mathfrak{R}\left(\frac{f^{\prime}(\mathrm{i} \omega)}{f(\mathrm{i} \omega)}\right) \mathrm{i} \omega$ 
stays always in the intervals $\left((n-2 \mathcal{N}-1) \frac{\pi}{2},(n-2 \mathcal{N}+\right.$ 1) $\frac{\pi}{2}$ ), where $\mathcal{N}=0,1,2,3, \ldots$. These intervals have no overlaps to each other for different value of $\mathcal{N}$. If there are two real numbers $\bar{R}, \underline{R}$ with $\underline{R}<\bar{R}$, a positive integer $\mathcal{N}$, and sufficiently large $T>0$, such that

$$
\left\{\begin{array}{l}
\sup _{\substack{\Re(\lambda)>0 \\
|\lambda| \rightarrow \infty}}\left|\alpha_{0}\left(\mathrm{e}^{-(\underline{R}+\lambda) \tau_{1}}, \ldots, \mathrm{e}^{-(\underline{R}+\lambda) \tau_{m}}\right)\right|<1 \\
\int_{0}^{T} \Re\left(\frac{f^{\prime}(\underline{R}+\mathrm{i} \omega)}{f(\underline{R}+\mathrm{i} \omega)}\right) \mathrm{d} \omega<\frac{(n-2 \mathcal{N}-1) \pi}{2} \\
\frac{(n-2 \mathcal{N}-1) \pi}{2}<\int_{0}^{T} \Re\left(\frac{f^{\prime}(\bar{R}+\mathrm{i} \omega)}{f(\bar{R}+\mathrm{i} \omega)}\right) \mathrm{d} \omega
\end{array}\right.
$$

then there is at least one characteristic root $\lambda$ that stays on the line $\Re(\lambda)=\zeta$ with $\zeta \in(\underline{R}, \bar{R})$, and the number of characteristic roots that stay in $\Re(\lambda)>\zeta$ is $\mathcal{N}$. With this fact in mind, the characteristic roots of a given NDDE in a given domain of the complex plane can be numerically found out one by one, by searching all possible lines $\Re(\lambda)=\zeta$ with the above criteria for some $\mathcal{N}=0,1,2,3,4, \ldots$ In particular, the case $\mathcal{N}=0$ determines the rightmost characteristic $\operatorname{root}(\mathrm{s})$.

\section{Two illustrative examples}

Example 1 Consider the following linear NDDE arising from modeling real-time dynamic substructuring [6]

$\ddot{x}(t)+2 \xi \dot{x}(t)+x(t)+p \ddot{x}(t-\tau)=0$

whose characteristic equation for the trivial solution $x=0$ takes the form $f(\lambda)=0$, where

$f(\lambda)=\lambda^{2}+2 \xi \lambda+1+p \lambda^{2} \mathrm{e}^{-\lambda \tau}$

Accordingly, the function $W(\lambda)$ is

$W(\lambda)=\frac{f(\lambda)}{1+p \mathrm{e}^{-\lambda \tau}}$

Figure 2 presents the plot of $\int_{0}^{50.0} \Re\left(\frac{W^{\prime}(\mathrm{i} \omega)}{W(\mathrm{i} \omega)}\right) \mathrm{d} \omega$, an estimation of the testing integral, with respect to $\tau$ within $[0,8]$ for the NDDE when $\xi=0.05, p=0.2$. A similar plot is given in Fig. 3 by using the characteristic function $f(\lambda)$ directly, which is in agreement with Fig. 2. The two plots show that both at $\tau=\tau_{1}^{*} \approx 3.27$ and $\tau=\tau_{2}^{*} \approx 6.17$, a stability switch occurs. The solution $x=0$ is asymptotically stable if $\tau \in\left[0, \tau_{1}^{*}\right) \cup\left(\tau_{2}^{*}, 8\right]$ and is unstable if $\tau \in\left(\tau_{1}^{*}, \tau_{2}^{*}\right)$.

In particular, when $\tau=1$, one has

$$
\int_{0}^{50.0} \Re\left(\frac{W^{\prime}(\mathrm{i} \omega)}{W(\mathrm{i} \omega)}\right) \mathrm{d} \omega \approx 3.1399>\frac{(2-1) \pi}{2}
$$

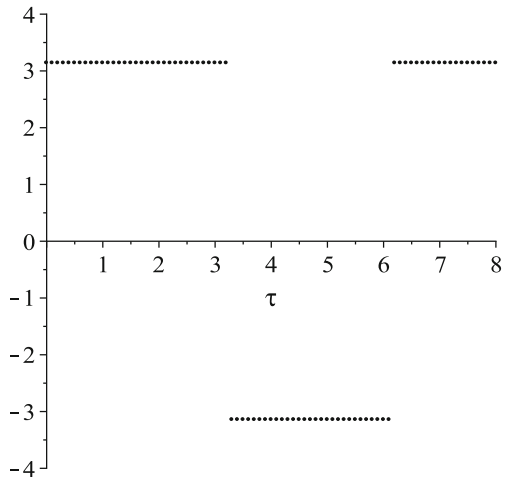

Fig. 2 The plot of the testing integral $\int_{0}^{50.0} \Re\left(\frac{W^{\prime}(\mathrm{i} \omega)}{W(\mathrm{i} \omega)}\right) \mathrm{d} \omega$ vs $\tau$ for Eq. (24) with $\xi=0.05, p=0.2$

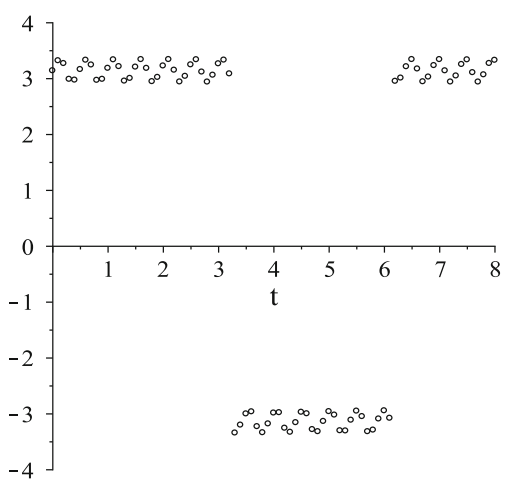

Fig. 3 The plot of the testing integral $\int_{0}^{50.0} \Re\left(\frac{f^{\prime}(\mathrm{i} \omega)}{f(\mathrm{i} \omega)}\right) \mathrm{d} \omega$ vs $\tau$ for Eq. (24) with $\xi=0.05, p=0.2$

which implies that $\sigma<0$. Moreover, one has $\sup _{\substack{\Re(\lambda)>0 \\|\lambda| \rightarrow \infty \\ \infty}}\left|\alpha_{0}\left(\mathrm{e}^{-(-0.2+\lambda) \tau}\right)\right|=0.2 e^{-(-0.2)} \approx 0.2443<1$

$$
\begin{aligned}
& \int_{0}^{50.0} \Re\left(\frac{W^{\prime}(-0.1+\mathrm{i} \omega)}{W(-0.1+\mathrm{i} \omega)}\right) \mathrm{d} \omega \approx 3.1917>\frac{(2-1) \pi}{2} \\
& \int_{0}^{50.0} \Re\left(\frac{W^{\prime}(-0.2+\mathrm{i} \omega)}{W(-0.2+\mathrm{i} \omega)}\right) \mathrm{d} \omega \approx-3.0834<\frac{(2-1) \pi}{2}
\end{aligned}
$$

Thus, $\sigma \in(-0.2,-0.1)$. With a freely chosen initial guess $-0.15 \in(-0.2,-0.1)$ for $\sigma$, we see from Fig. 4 that the imaginary part of the rightmost characteristic root(s) is close to 1 . Thus, starting from $-0.15+\mathrm{i}$, using Newton-Raphson's iteration method finds the the rightmost characteristic root(s) to be $-0.1155 \pm 0.9221 \mathrm{i}$, which is the same as the one obtained in [20].

Example 2 In order to reduce the vibration of a SDOF vibration system with mass $m$, damping coefficient $c$, elastic coefficient $k$ and harmonic excitation $f(t)$, a resonator can be installed for total absorption. With $m_{a}, c_{a}, k_{a}$ standing for 


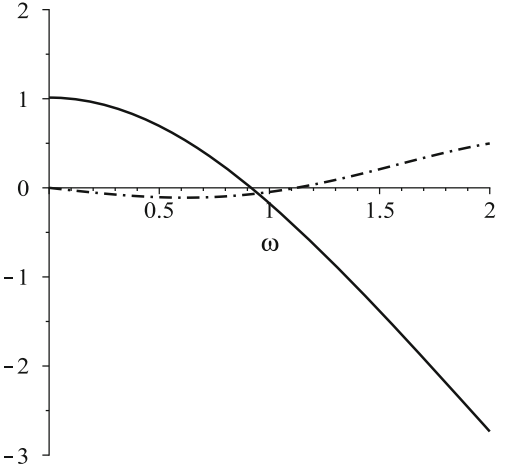

Fig. 4 The real and imaginary parts of $f(-0.15+\mathrm{i} \omega)$ vs $\omega$ for Eq. (24) with $\xi=0.05, p=0.2$ and $\tau=1$. At about $\omega \approx 1$, one has $f(-0.15+\mathrm{i} \omega) \approx 0$. Solid The real part; Dashed The imaginary part

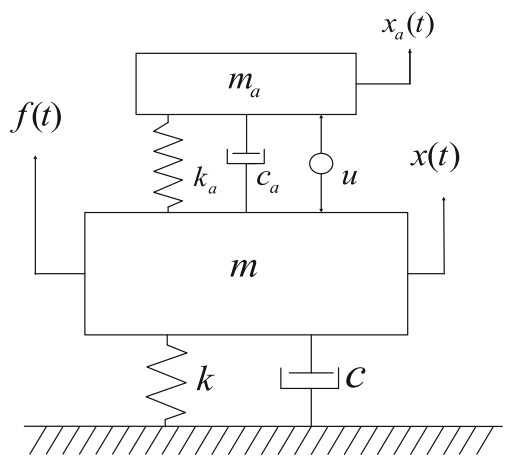

Fig. 5 The resonator based on the delayed feedback

the mass, damping coefficient, elastic coefficient of the resonator respectively, the combined resonator system can be described by

$\left\{\begin{array}{l}m_{a} \ddot{x}_{a}(t)+c_{a} \dot{x}_{a}(t)+k_{a} x_{a}(t)-c_{a} \dot{x}(t)-k_{a} x(t)=u \\ m \ddot{x}(t)+\left(c_{a}+c\right) \dot{x}(t)+\left(k_{a}+k\right) x(t)-c_{a} \dot{x}_{a}(t) \\ \quad-k_{a} x_{a}(t)=-u+f(t)\end{array}\right.$

where $u$ is a feedback control to the resonator, see Fig. 5 . When total absorption occurs, namely $x(t) \rightarrow 0$ as $t \rightarrow$ $+\infty$, one has

$\left\{\begin{array}{l}m_{a} \ddot{x}_{a}(t)+c_{a} \dot{x}_{a}(t)+k_{a} x_{a}(t)-u=0 \\ -c_{a} \dot{x}_{a}(t)-k_{a} x_{a}(t)+u=f(t)\end{array}\right.$

It follows that $m_{a} \ddot{x}_{a}(t)=f(t)$. If $f(t)$ takes simply as $f(t)=f_{0} \sin \left(\omega_{0} t\right)$, then under the zero initial condition, one has $x_{a}(t)=f_{0} \sin \left(\omega_{0} t\right) /\left(m_{a} \omega_{0}^{2}\right)$.

When $u=-g \ddot{x}_{a}(t-\tau)$, the characteristic equation of the resonator is given by

$f(\lambda) \equiv m_{a} \lambda^{2}+c_{a} \lambda+k_{a}+g \lambda^{2} \mathrm{e}^{-\lambda \tau}=0$

In order that the resonator vibrates with frequency $\omega_{0}$, it requires that Eq. (28) has a pair of conjugate roots $\lambda= \pm i \omega_{0}$, the feedback gain $g$ is determined from $f\left(\mathrm{i} \omega_{0}\right)=0$.

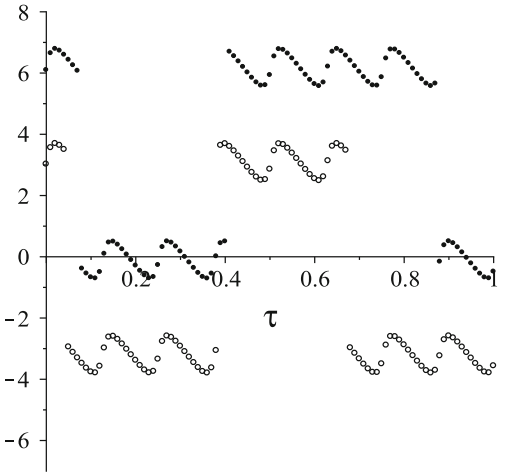

Fig. 6 The plot of testing integral of $\int_{0}^{50.0} \Re\left(\frac{F^{\prime}(\mathrm{i} \omega)}{F(\mathrm{i} \omega)}\right) \mathrm{d} \omega$ and $\int_{0}^{50.0} \Re\left(\frac{f^{\prime}(\mathrm{i} \omega)}{f(\mathrm{i} \omega)}\right) \mathrm{d} \omega$ vs $\tau$ for Eq. (26) and Eq. (27), with $m=1 \mathrm{~kg}$, $c=2 \mathrm{~kg} / \mathrm{s}, k=100 \mathrm{~N} / m, m_{a}=0.2 \mathrm{~kg} c_{a}=0.5 \mathrm{~kg} / \mathrm{s}, k_{a}=10 \mathrm{~N} / \mathrm{m}$, $\omega_{0}=10 \mathrm{rad} / \mathrm{s} g=-0.1118 \mathrm{~N} / \mathrm{m}$. Points for $F(\lambda)$; Circles for $f(\lambda)$

Moreover, the characteristic equation of the combined resonator system is given by $F(\lambda) \equiv \operatorname{det}(\Lambda)=0$, where

$\Lambda=\left[\begin{array}{cc}f(\lambda), & -c_{a} \lambda-k_{a} \\ -c_{a} \lambda-k_{a}-g \lambda^{2} \mathrm{e}^{-\lambda \tau}, & m \lambda^{2}+\left(c_{a}+c\right) \lambda+\left(k_{a}+k\right)\end{array}\right]$

Hence, the stability of the combined resonator system can be carried out as done in Example 1. Here, using the characteristic function $F(\lambda)$ directly is preferable.

In particular, when $m=1 \mathrm{~kg}, c=2 \mathrm{~kg} / \mathrm{s}, k=100 \mathrm{~N} / \mathrm{m}$, $m_{a}=0.2 \mathrm{~kg}, c_{a}=0.5 \mathrm{~kg} / \mathrm{s}, k_{a}=10 \mathrm{~N} / \mathrm{m}, \omega_{0}=10 \mathrm{rad} / \mathrm{s}$ one finds $g=-0.1118 \mathrm{~N} / \mathrm{m}$ Taking $\tau$ as the parameter, the plot of the test integral for $f(\lambda)$ and $F(\lambda)$ with respect to the delay $\tau$ is shown in Fig. 6 for $T=50$. It shows that for the combined resonator system the critical delay values within $\tau \in[0,1]$ are found to be

$\tau_{1}^{*} \approx 0.078, \tau_{2}^{*} \approx 0.4, \tau_{3}^{*} \approx 0.875$

thus the solution $x=0$ of the combined system is asymptotically stable if $\tau \in\left[0, \tau_{1}^{*}\right) \cup\left(\tau_{2}^{*}, \tau_{3}^{*}\right)$ and unstable if $\tau \in\left(\tau_{1}^{*}, \tau_{2}^{*}\right) \cup\left(\tau_{3}^{*}, 1\right]$; while for the resonator only, the critical delay values within $\tau \in[0,1]$ are found to be

$\tau_{1}^{* *} \approx 0.046, \tau_{2}^{* *} \approx 0.382, \tau_{3}^{* *} \approx 0.675$

and the solution $x=0$ of the resonator is asymptotically stable if $\tau \in\left[0, \tau_{1}^{* *}\right) \cup\left(\tau_{2}^{* *}, \tau_{3}^{* *}\right)$ and unstable if $\tau \in\left(\tau_{1}^{* *}, \tau_{2}^{*}\right) \cup\left(\tau_{3}^{* *}, 1\right]$. Here $\tau_{1}^{* *} \in\left(0, \tau_{1}^{*}\right), \tau_{3}^{* *} \in\left(\tau_{2}^{*}, \tau_{3}^{*}\right)$, both stay in the stable intervals of the combined system, and at the critical delays $\tau \approx \tau_{1}^{* *}$ and $\tau \approx \tau_{3}^{* *}$, the characteristic function $f(\lambda)$ has no pure imaginary roots except $\pm \mathrm{i} \omega_{0}$, thus the resonator vibrates with the same frequency $\omega_{0}$ of the excitation, but the vibration of the main part with mass $m$ of the combined system is totally reduced to zero due to resonance.

In the following, the rightmost characteristic $\operatorname{root}(\mathrm{s})$ of the combined system will be calculated for $\tau=\tau_{1}^{* *}$ and $\tau=\tau_{3}^{* *}$. 


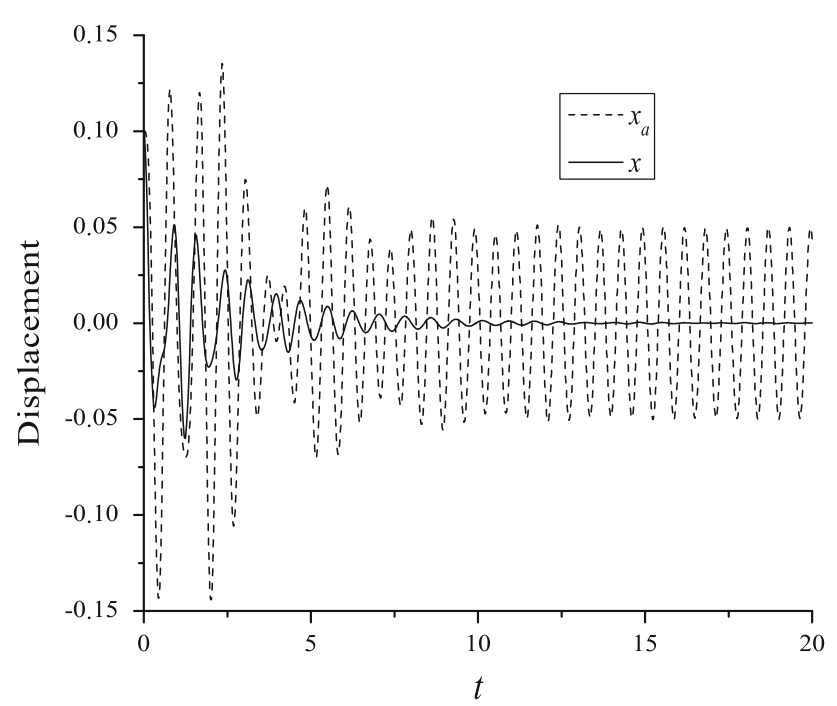

Fig. 7 The displacement of Eq.(26) with $m=1 \mathrm{~kg}, c=2 \mathrm{~kg} / \mathrm{s}$, $k=100 \mathrm{~N} / \mathrm{m}, m_{a}=0.2 \mathrm{~kg}, c_{a}=0.5 \mathrm{~kg} / \mathrm{s}, k_{a}=10 \mathrm{~N} / \mathrm{m}$, $\omega_{0}=10 \mathrm{rad} / \mathrm{s}, g=-0.1118 \mathrm{~N} / \mathrm{m}$, and $\tau=\tau_{1}^{* *} . x$ : displacement of $m ; x_{a}$ : displacement of $m_{a}$

For the two cases, $\alpha<0$, thus, $\underline{R}$ and $\bar{R}$ should be chosen from negative numbers.

For $\tau=\tau_{1}^{* *}=0.046$, one has,

$\sup _{\substack{\Re(\lambda)>0 \\|\lambda| \rightarrow \infty}}\left|\alpha_{0}\left(\mathrm{e}^{-(-0.4+\lambda) \tau_{1}^{* *}}\right)\right|=\left|\frac{g}{m_{a}} \mathrm{e}^{0.4 \tau_{1}^{* *}}\right| \approx 0.5694<1$

$$
\begin{aligned}
& \int_{0}^{200.0} \Re\left(\frac{W^{\prime}(-0.2+\mathrm{i} \omega)}{W(-0.2+\mathrm{i} \omega)}\right) \mathrm{d} \omega \approx 6.2676>\frac{(4-1) \pi}{2} \\
& \int_{0}^{200.0} \Re\left(\frac{W^{\prime}(-0.4+\mathrm{i} \omega)}{W(-0.4+\mathrm{i} \omega)}\right) \mathrm{d} \omega \approx-0.0115<\frac{(4-1) \pi}{2}
\end{aligned}
$$

Thus, $\alpha \in(-0.4,-0.2)$. With $\alpha_{0}=-0.3 \in(-0.4,-0.2)$ as an initial guess, $F(-0.3+8 \omega) \approx 0$ gives an estimation $\omega_{0} \approx 11$ of $\omega$, then using Newton-Raphson's iteration method finds the rightmost characteristic roots are $-0.3765 \pm$ 8.1050i. As for $\tau=\tau_{3}^{* *}=0.675$, the same procedure above finds the rightmost characteristic roots $-0.3929 \pm 10.8767 \mathrm{i}$. The results can be confirmed by using a matlab-based package DDE-BIFTOOL [21]. As shown in Figs. 7 and 8, for both $\tau=\tau_{1}^{* *}=0.046$ and $\tau=\tau_{3}^{* *}=0.675$, the vibration of the main part of the combined system has been totally reduced.

\section{A graphical stability criterion}

As seen above, the proposed stability criteria does work effectively in the stability analysis of NDDEs. These criteria can also be used to derive a graphical stability criterion, which enables us to determine the stability of a given NDDE graphically. In fact, for a given constant $c>0$, let

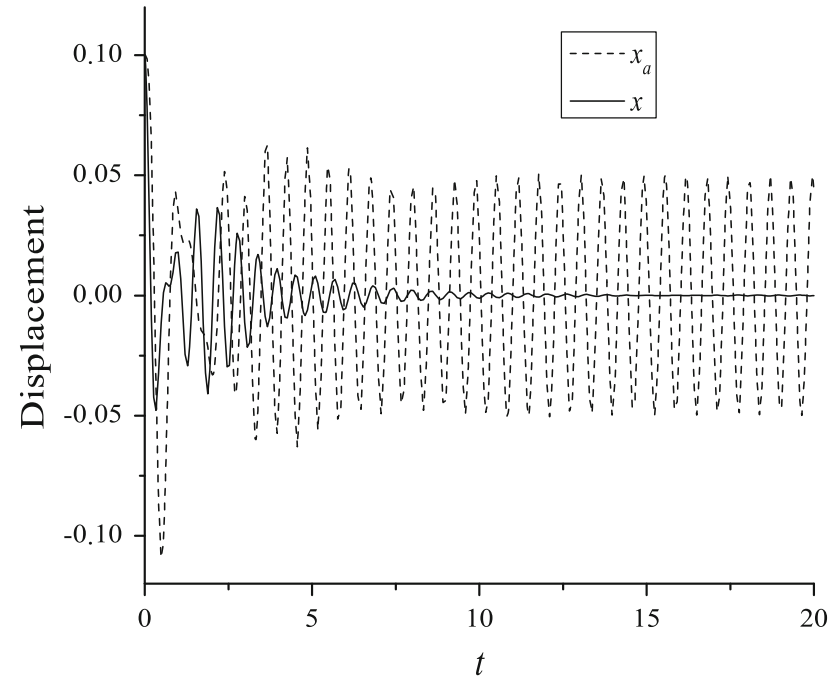

Fig. 8 The displacement of Eq. (26) with $m=1 \mathrm{~kg}, c=2 \mathrm{~kg} / \mathrm{s}$, $k=100 \mathrm{~N} / \mathrm{m}, m_{a}=0.2 \mathrm{~kg}, c_{a}=0.5 \mathrm{~kg} / \mathrm{s}, k_{a}=10 \mathrm{~N} / \mathrm{m}, \omega_{0}=$ $10 \mathrm{rad} / \mathrm{s}, g=-0.1118 \mathrm{~N} / \mathrm{m}$ and $\tau=\tau_{3}^{* *} . x$ : displacement of $m ; x_{a}$ : displacement of $m_{a}$

$\Pi(\lambda)=\frac{f(\lambda)}{(\lambda+c)^{n}}$

where $f(\lambda)$ is the characteristic function defined by (2) subject to (3). Moreover, let

$\Pi(\mathrm{i} \omega)=P(\omega)+\mathrm{i} Q(\omega)$

Then we have

Theorem 3 Assume that the conditions of Theorem 1 hold, then $\mathcal{N}=0$ if there is a constant $c>0$ such that the Nyquist frequency plot, defined by the curve of

$\{(P(\omega), Q(\omega)):-\infty<\omega<+\infty\}$

does not encircle the origin of the complex plane.

Proof The argument rule implies that

$\arg \Pi(\mathrm{i} \omega)=\arg f(\mathrm{i} \omega)-\arg \left((\mathrm{i} \omega+c)^{n}\right)$

then one has,

$\left.\arg \Pi(\mathrm{i} \omega)\right|_{0} ^{T}=\left.\arg (f(\mathrm{i} \omega))\right|_{\omega=0} ^{\omega=T}-\left.\arg \left((\mathrm{i} \omega+c)^{n}\right)\right|_{\omega=0} ^{\omega=T}$

With a fixed $c>0$, it holds

$\left.\lim _{T \rightarrow+\infty} \arg \left((\mathrm{i} \omega+c)^{n}\right)\right|_{\omega=0} ^{\omega=T}=\frac{n \pi}{2}$

Thus, according to Lemma $1, \mathcal{N}=0$ if and only if there is a $T_{0}>0$ such that for all $T>T_{0}$ one has

$\left.\arg \Pi(\mathrm{i} \omega)\right|_{0} ^{T} \in\left(\frac{-\pi}{2}, \frac{\pi}{2}\right)$

Note that under the assumption (3), for sufficiently large $\omega>0$, one has $P(\omega)>0$. It means that the Nyquist frequency plot must eventually stay in the right-half complex 


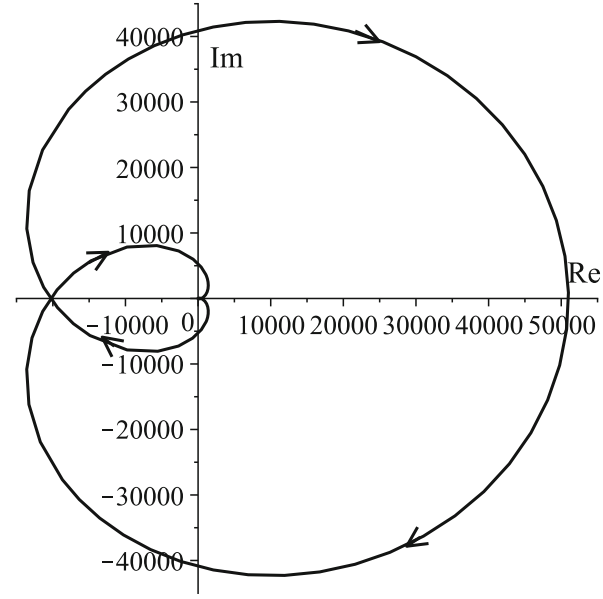

(a) $\{\Pi(\mathrm{i} \omega): \omega \in(-\infty,+\infty)\}$

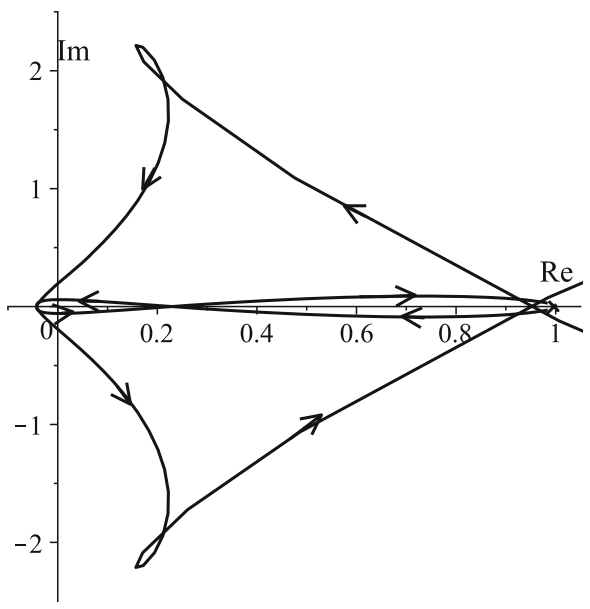

(b) zoom around the origin

Fig. 9 The Nyquist frequency plot of $\Pi(\mathrm{i} \omega)$ with $c=1$

plane. Since the Nyquist frequency plot is asymmetric to the real axis, thus, (31) is true if there is a constant $c>0$ such that the Nyquist frequency plot does not encircle the origin of the complex plane. This completes the proof.

With this graphical stability criterion, it is not required to know the exact curve of the Nyquist plot, but just to know whether the Nyquist plot encircles the origin or not. It should be pointed out that with fixed $c=1$ as done in $[8,10]$, the Nyquist frequency plot may not help in stability test in some cases.

Example 3 Let us consider the characteristic function of a six-order RDDE, described by

$$
\begin{aligned}
f(\lambda)= & \lambda^{6}+1.5 \lambda^{5}+126 \lambda^{4}+100 \lambda^{3}+4381 \lambda^{2} \\
& +938 \lambda+31250+\left(1.5 \lambda^{5}+23 \lambda^{4}+130 \lambda^{3}\right. \\
& \left.+1619 \lambda^{2}+1890 \lambda+19750\right) \mathrm{e}^{-\lambda \tau}
\end{aligned}
$$

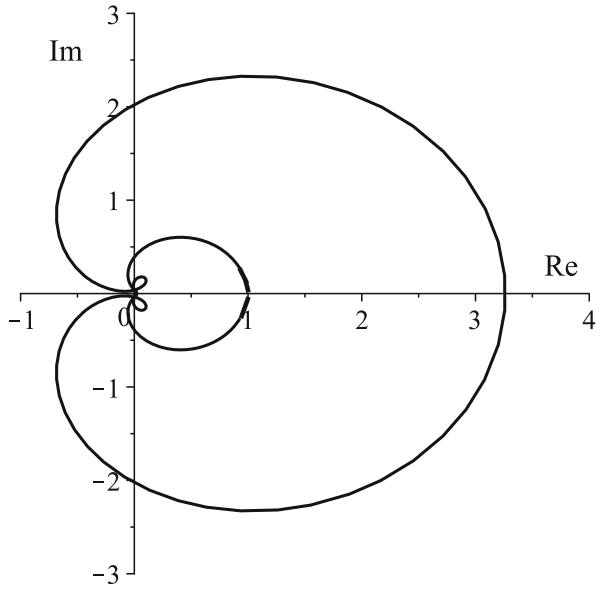

(a) $\{\Pi(\mathrm{i} \omega): \omega \in(-\infty,+\infty)\}$

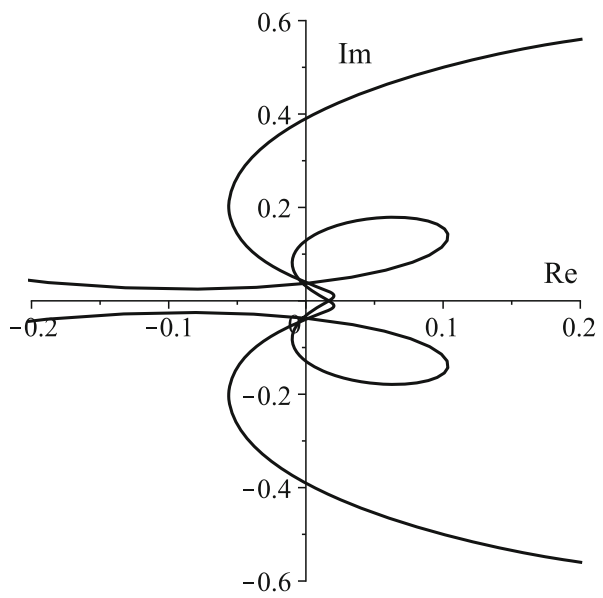

(b) zoom around the origin

Fig. 10 The Nyquist frequency plot of $\Pi(\mathrm{i} \omega)$ with $c=5$

When $\tau=0.01$, straightforward calculation gives,

$$
\int_{0}^{100.0} \Re\left(\frac{f^{\prime}(\mathrm{i} \omega)}{f(\mathrm{i} \omega)}\right) \mathrm{d} \omega=9.4032>\frac{(6-1) \pi}{2}
$$

It means that $\mathcal{N}=0$. To check this fact graphically, let

$\Pi(\lambda)=\frac{f(\lambda)}{(\lambda+c)^{6}}$

With $c=1$, the Nyquist frequency plot of $\Pi(\lambda)$ is shown in Fig. 9, where the arrow indicates the direction of the Nyquist plot as $\omega$ increases. A detailed calculation shows that when $\omega$ increases from $-\infty$ to $+\infty$, the total change of the argument $\arg (\Pi(\mathrm{i} \omega)$ equals zero, this is in agreement with the fact $\mathcal{N}=0$. In this case, the Nyquist plot does not help much in judging the stability. However, if we take $c=5$, then as shown obviously in Fig. 10, the Nyquist plot does not encircle the origin. This fact justifies $\mathcal{N}=0$ directly. 
Similar cases occur for NDDEs. Thus we must be cautious in judging the stability when the Nyquist plot encircles the origin of the complex plane.

\section{Conclusions}

In this paper, three Mikhailov-type stability criteria are established for the stability test of a class of time-invariant NDDEs. Two of them (Theorem 1; Corollary 1) are expressed in terms of an axillary function associated with the characteristic function, and the other (Theorem 2) is represented in terms of the characteristic function directly. The peculiarity of these criteria is that in stability testing, it is not required to calculate the exact value of the testing integral defined over infinite integrating interval, but it just requires to calculate a rough estimation of the testing integral over a considerable short interval. With the well-developed numerical methods for integration, the stability test can be carried out easily. The three criteria are mathematically equivalent to each other, but using Theorem 2 is more preferable, because it uses the characteristic function directly and it has much less computational complexity than using the auxiliary function. Based on the proposed stability criteria, a three-step numerical scheme is proposed for calculating the rightmost root(s) of NDDEs, each step is easy for numerical implementation. This scheme works also for calculating other characteristic roots. Numerical examples show that the proposed method works effectively, but a schematic routine for finding an estimation of the testing integral is left for future consideration. The graphical stability criterion (Theorem 3 ) is a sufficient condition for the asymptotical stability of a given NDDE and it can be used in a more intuitive way than numerical calculation.

Acknowledgments This work was supported by NSF of China under Grants 11372354 and 11032009, and by the Priority Academic Program Development of Jiangsu Higher Education Institutions.

\section{References}

1. Kuang Y (1993) Delay differential equations with applications to population dynamics. Academic Press, New York

2. Niculescu SI (2001) Delay effects on stability: a robust control approach. Springer, London

3. Olgac N, Jalili N (1998) Modal analysis of flexible beams with delayed resonator vibration absorber: theory and experiments. $\mathrm{J}$ Sound Vib 218:307-331

4. Masoud ZN, Nayfeh AH (2003) Sway reduction on container cranes using delayed feedback controller. Nonlinear Dyn 34:347358
5. Nayfeh AH, Masoud ZN, Nayfeh NA (2011) A smart sway controller for cranes from theory to laboratory to industry. Vibration problems ICoVP 2011 Supplement. Technical University of Liberec, Liberec, pp 14-29

6. Kyrychko YN, Blyuss KB, Gonzalez-Buelga A, Hogan SJ, Wagg DJ (2006) Real-time dynamic substructuring in a coupled oscillatorCpendulum system. Proc R Soc A 462:1271-1294

7. Barton DAW, Krauskopf B, Wilson RE (2007) Homoclinic bifurcations in a neutral delay model of a transmission line oscillator. Nonlinearity 20:809-829

8. Fu MY, Olbrot AW, Polis MP (1989) Robust stability for time-delay systems: the edge theorem and graphical tests. IEEE Trans Autom Control 34:813-820

9. Buslowicz M (2008) Stability of linear continuous-time fractional order systems with delays of the retarded type. Bull Pol Acad Sci Tech Sci 56:319-324

10. Fu MY, Olbrot AW, Polis MP (1991) The edge theorem and graphical tests for robust stability of neutral time-delay systems. Automatica 27:739-741

11. Hassard B (1997) Counting roots of the characteristic equations for linear delay-differential equations. J Differ Equ 136:222-235

12. Stepan G (1989) Retarded dynamical systems: stability and characteristic functions. Longman Scientific \& Technical, New York

13. Li JY (2010) Study on stability and dynamic response of time delay systems with complex coefficients. Dissertation, Nanjing University of Aeronautics and Astronautics

14. Kolmanovskii VB, Nosov VR (1986) Stability of functional differential equations. Academic Press, London

15. Rifat $\mathrm{S}$, Olgac $\mathrm{N}$ (2006) Complete stability analysis of neutral-type first order two-time-delay systems with cross-talking delays. SIAM J Control Optim 45:957-971

16. Insperger T, Stepan G (2002) Semi-discretization method for delayed systems. Int J Numer Method Eng 55:503-518

17. Insperger T, Stepan G, Janos T (2010) Delayed feedback of sampled higher derivatives. Philos Trans R Soc A 368:469-482

18. Insperger T, Stepan G (2011) Semi-discretization for time-delay systems. Springer, London

19. Wang ZH, Hu HY (2008) Calculation of the rightmost characteristic root of retarded time-delay systems via Lambert $\mathrm{W}$ function. J Sound Vib 318:757-767

20. Wang ZH (2008) Numerical stability test of neutral delay differential equations. Math Probl Eng. doi:10.1155/698043

21. Engelborghs K, Luzyanina T, Samaey G (2000) DDE-BIFTOOL: a Matlab package for bifurcation analysis of delay differential equations. TW, Report 305

22. Barton DAW, Krauskopf B, Wilson RE (2006) Collocation schemes for periodic solutions of neutral delay differential equations. J Differ Equ App 12:1087-1101

23. Kolmanovskii V, Myshkis A (1999) Introduction to the Theory and Applications of Functional Differential Equations. Kluwer, Dordrecht

24. Wang ZH, Du ML, Shi M (2011) Stability test of fractional-delay systems via integration. Sci China Phys. Mech. \& Astr. 54:18391846 